\title{
Bibliometric Analysis of Social Science Based on ESI and InCites-Taking Huazhong University of Science and Technology as an Example
}

\author{
Wei Fengping, Fang Ji*, Huang Jin, He Yihua, Zhang Rong \\ Library of Huazhong University of Science and Technology \\ Wuhan 430074 \\ Email: fangji@hust.edu.cn
}

\begin{abstract}
With the subject data in the field of ESI and InCites social science as the research object, a comparison was made in this paper between the standard institutions (including Tsinghua University, Shanghai Jiaotong University, Zhejiang University and Xi'an Jiaotong University) and Huazhong University of Science and Technology in the article number, citation frequency, research theme, journal, etc. by bibliometric method to analyze the gaps and deficiencies of Huazhong University of Science and Technology and finally put forward the opinions and suggestions.
\end{abstract}

Keywords-Social science; Bibliometrics; ESI; InCites

\section{INTRODUCTION}

ESI [1] and InCites [2], as the analysis and evaluation databases launched by Clarivate Analytics in the United States, are often used to measure the scientific research performance of institutions and track the trend of scientific development. ESI can analyze and evaluate the papers, journals, and institutions from a global perspective in 22 subject fields. InCites can provide basic paper data and measurement indicators, and the subject analysis combining both of them can provide more deep thinking and more effective countermeasures for subject construction [3].

Huazhong University of Science and Technology is a comprehensive and research-oriented university, there are 15 subject fields including social science having ranked the top $1 \%$ of ESI Global, among which engineering science, material science and computer science have ranked the top 1\%o. A comparison was made in the text between the universities with the same outstanding science and technology advantages including Tsinghua University, Shanghai Jiaotong University, Zhejiang University and Xi'an Jiaotong University selected herein as benchmarking institutions and Huazhong University of Science and Technology, in order to analyze the features and deficiencies of Huazhong University of Science and Technology in social science, so as to provide references for the scientific construction and development

\section{DATA AND METHODS}

\section{A. Data source}

The indicator data of the institutions were captured in the "Social science, general" field from the ESI database with the time coverage range of the indicator data updated in January 2018 from January 2007 to October 2017. The "institution" and "journal" analysis modules of InCites database were used, and "Social science, general" under ESI was selected as the research direction, the Article and Review were selected as the reference type, and the time range was set from 2007 to 2017, making the InCites indicator data as close as possible to ESI indicator data.

The collection and statistics of the exported indicator data were carried out by using Excel, some of the data were directly processed by using the InCites database, and the data retrieval time was February 27, 2018.

\section{B. Bibliometric Indexes and Research Methods}

Tsinghua University, Shanghai Jiaotong University, Zhejiang University and Xi'an Jiaotong University were selected as benchmarking institutions, the differences in the paper number, citation frequency and other indicators were compared and analyzed, and the characteristics of the development of social sciences in Huazhong University of Science and Technology were summarized.

Bibliometric indicators and their significance: (1) Papers, number of web of science documents, in this article only article and review are discussed; (2) Cites, number of times the set of publications has been cited;(3)Cites per paper, the ratio of the cites to papers; (4) $\mathrm{HCP}$ (Highly Cited Papers), a paper that belongs to the top $1 \%$ of papers in a research field published in a specified year, the $1 \%$ is determined by the highly cited threshold calculated for the research field in the specified year;

(5)HP(Hot Papers), a paper published in the past two years that received a number of citations in the most recent two-month period that places it in the top $0.1 \%$ of papers in the same field; 
(6) Inter collaborat (International collaborations), papers that contain one or more international co-author; (7) \% Inter collaborat (\% International collaborations), percentage of publications that have international co-authors; (8) CNCI (Category Normalized Citation Impact),Citation impact (citations per paper) normalized for subject, year and document type.

\section{RESULTS AND DISCUSSIONS}

\section{A. General Situations}

In the ranking data of the current issue of ESI, there were 1,382 institutions in the social sciences fields, including 30 in mainland China. Tsinghua University ranked No. 441, Shanghai Jiaotong University, Zhejiang University and Huazhong University of Science and Technology were located between No. 501 and No. 1000, and Xi'an Jiaotong University was located after No. 1000, as shown in Table 1.
Tsinghua University ranks the first in the cited frequency and cited papers. Shanghai Jiaotong University ranks the first in the cites per paper and inter collaborats, and more than $55 \%$ of the papers are from inter collaborats. Zhejiang University ranks the first in the number of papers and inter collaborates, but the last in the cites per paper. Huazhong University of Science and Technology ranks the last in the number and percentage of inter collaborats, and the cites per paper is relatively low. There are more than ten highly cited papers in Tsinghua University, Shanghai Jiaotong University and Zhejiang University, and there are less than ten highly cited papers in Huazhong University of Science and Technology and Xi'an Jiaotong University. Except for Xi'an Jiaotong University without hot papers, and all the other four universities have a hot paper.

Compared with Tsinghua University and Zhejiang University, the number of papers in Huazhong University of Science and Technology and Xi'an Jiaotong University is relatively low.

TABLE I. GENERAL SITUATIONS OF THE FIVE UNIVERSITIES IN SOCIAL SCIENCES

\begin{tabular}{ccccccccc}
\hline Inst & ESI Rank. & Doc & Cites & Cites per paper & HCP & HP & Inter Collaborat & \% Inter Collaborat \\
\hline THU & 441 & 810 & 6425 & 7.93 & 18 & 1 & 365 & 43.76 \\
SJTU & 517 & 602 & 5367 & 8.92 & 14 & 1 & 340 & 55.11 \\
ZJU & 567 & 813 & 4751 & 5.84 & 13 & 1 & 395 & 47.48 \\
HUST & 864 & 422 & 2593 & 6.14 & 8 & 1 & 158 & 36.24 \\
XJTU & 1025 & 305 & 2040 & 6.69 & 5 & 0 & 171 & 52.78
\end{tabular}

\section{B. Analysis of Output of the Papers over the Years}

From 2003 to 2013, the paper in the field of social sciences in China's colleges and universities presented a pattern of rapid growth year by year [4]. From 2007 to 2017, the output of the Five-School Papers were not increased year by year, and the fluctuant change was displayed, but a growth development trend was still showed in general, as shown in Figure 1.

Tsinghua University showed a steady growth from 2007 to 2012, and the number of its papers was most advantageous. It was declined slightly in 2013 and then entered into rapid growth again in 2015. It has been grown faster in Zhejiang
University since 2011, surpassing Shanghai Jiaotong University and having been very close to or surpassed Tsinghua University. There have been fewer papers in Shanghai Jiaotong University than Tsinghua University and Zhejiang University since 2011. It was progressed greatly in Huazhong University of Science and Technology in 2013, and developed more slowly in the rest of the year. The number of papers was grown slowly in Xi'an Jiaotong University, and it was apparently declined in 2016, but its growth rate in 2017 was relatively large, almost catching up with Huazhong University of Science and Technology.

TABLE II. CHANGES IN PAPER OUTPUT OF THE FIVE UNIVERSITIES FROM 2007 TO 2017

\begin{tabular}{cccccccccccc}
\hline INST & 2007 & 2008 & 2009 & 2010 & 2011 & 2012 & 2013 & 2014 & 2015 & 2016 & 2017 \\
\hline THU & 21 & 37 & 46 & 56 & 72 & 85 & 79 & 84 & 103 & 118 & 133 \\
SJTU & 19 & 26 & 38 & 47 & 43 & 48 & 65 & 71 & 94 & 85 & 81 \\
ZJU & 13 & 17 & 39 & 32 & 71 & 70 & 80 & 124 & 122 & 114 & 150 \\
HUST & 10 & 13 & 16 & 25 & 23 & 28 & 54 & 60 & 66 & 74 & 67 \\
XJTU & 7 & 9 & 19 & 28 & 20 & 30 & 30 & 36 & 44 & 33 & 68 \\
\hline
\end{tabular}




\section{Journal Distribution analysis}

There are more than 1900 journals in the field of ESI social science [5], the vast majority of which are English journals. Some scholars believe that under the influence of cultural and linguistic factors, it is difficult for China's humanistic and social science research achievements to be fully reflected in international journals [6]. ESI journal coverage is relatively narrower. Among the five universities, there are the most kinds of journals distributed in the field of social sciences in Zhejiang University, i.e. 328 kinds, and there are also more than 300 kinds (up to 303 kinds) in Tsinghua University, 246 kinds in Shanghai Jiaotong University, 171 kinds in Huazhong University of Science and Technology, and more than 170 kinds in Xi'an Jiaotong University. The number of papers in Huazhong University of Science and Technology is more than 1 higher than Xi'an Jiaotong University, but there are only one more kind of journals.
The journals with more than ten papers issued in the five universities are shown in Table 2. Tsinghua University, Zhejiang University, Xi'an Jiaotong University, and Shanghai Jiaotong University have published more than 10 papers in the Energy Policy. Tsinghua University has published 127 papers in the journal which is the only journal with published paper volume of more than 100 papers. Tsinghua University is also the only university that has published more than 100 papers in the same type of journal. Huazhong University of Science and Technology, Zhejiang University, Shanghai Jiaotong University and Xi'an Jiaotong University have published more than 10 papers in BMC Public Health and PLOS One. Huazhong University of Science and Technology has the highest published paper volume in BMC Public Health, reaching 39 papers, and the journal is also one journal in which Huazhong University of Science and Technology has the highest published paper volume.

TABLE III. ANALYSIS OF PAPERS AND JOURNALS OF THE FIVE UNIVERSITIES IN SOCIAL SCIENCE

\begin{tabular}{|c|c|c|}
\hline Inst & & Journal Analysis \\
\hline THU & 303 & $\begin{array}{l}\text { Energy Policy(127); Accident Analysis And Prevention(20); Chinese Journal Of International } \\
\text { Politics(16); Transportation Research Part D-Transport And Environment(13); Social } \\
\text { Indicators Research(13); Information Processing \& Management(12); Research } \\
\text { Policy(11); Modern China(11); Climate Policy(11); Habitat International(10); Traffic Injury } \\
\text { Prevention(10). }\end{array}$ \\
\hline SJTU & 246 & $\begin{array}{l}\text { Cancer Epidemiology Biomarkers \& Prevention(67); American Journal Of } \\
\text { Epidemiology(36); BMC Public Health(16); Journal Of Contemporary China(16); Plos } \\
\text { One(14); Energy Policy(12); Transportation Research Part A-Policy And Practice(12). }\end{array}$ \\
\hline ZJU & 328 & $\begin{array}{l}\text { Habitat International(39); Scientometrics(27); BMC Public Health(17); Land Use } \\
\text { Policy(17); Journal Of Informetrics(16); Plos One(15); Energy Policy(14); Landscape And } \\
\text { Urban Planning(13); Cities(12); Semiotica(12); Applied Geography(10). }\end{array}$ \\
\hline HUST & 171 & $\begin{array}{l}\text { BMC Public Health(39); Plos One(20); Public Health(15); Scientometrics(12); American } \\
\text { Journal Of Epidemiology }(12) ; \text { International Journal Of Mobile Communications(10). }\end{array}$ \\
\hline XJTU & 170 & BMC Public Health (30); Energy Policy (14); Plos One (11). \\
\hline
\end{tabular}

\section{Research Theme Analysis}

The papers of the five universities in the social science field involve in multiple research themes. The papers of Tsinghua University in the social science field papers involve in 79 themes, and 81 in Shanghai Jiaotong University, 94 in Zhejiang University (maximum), 69 in Huazhong University of Science and Technology (minimum), and 77 in Xi'an Jiaotong University, as shown in Table 3.

The most frequent theme for the references in Shanghai Jiaotong University, Zhejiang University, Huazhong University of Science and Technology and Xi'an Jiaotong University is Public, Environmental \& Occupational Health, there are more than 200 papers with this theme in Shanghai Jiaotong University and Huazhong University of Science and Technology and more than 100 papers in Zhejiang University and Xi'an Jiaotong University. There are more than 100 papers under five themes having the most literature volume in Tsinghua University, there are up to 240 papers under the theme of Environmental Studies having the highest literature volume, and 79 papers under the theme of Public, Environmental \& Occupational Health, ranking the seventh. There are also more than 100 papers on Environmental Studies and Information Science \& Library Science in Zhejiang University.

The top five themes in the paper number include Information Science \& Library Science, and there are more than 100 papers in Zhejiang University and Tsinghua University. Environmental Studies is also included in the top five themes in the literature volume in Tsinghua University, Zhejiang University, Huazhong University of Science and Technology and Xi'an Jiaotong University, and there are 41 papers under this theme in Shanghai Jiaotong University, ranking the sixth. 
TABLE IV. RESEARCH THEMES OF THE FIVE UNIVERSITIES IN SOCIAL SCIENCE

\begin{tabular}{|c|c|c|}
\hline Inst & & Research Areas \\
\hline THU & 79 & $\begin{array}{c}\text { Environmental Studies (240); Economics (173); Environmental Sciences (133); Energy \& Fuels } \\
\text { (128); Information Science \& Library Science (100). }\end{array}$ \\
\hline SJTU & 81 & $\begin{array}{c}\text { Public, Environmental \& Occupational Health (255); Oncology (67); Transportation (63); Law (49); } \\
\text { Information Science \& Library Science (49). }\end{array}$ \\
\hline ZJU & 94 & $\begin{array}{l}\text { Public, Environmental \& Occupational Health (162); Environmental Studies (140); Information } \\
\text { Science \& Library Science (116); Urban Studies (83); Linguistics (68). }\end{array}$ \\
\hline HUST & 69 & $\begin{array}{c}\text { Public, Environmental \& Occupational Health(208);Information Science \& Library } \\
\text { Science(62);Health Policy \& Services(27);Environmental Studies(27);Social Sciences, } \\
\text { Interdisciplinary(23). }\end{array}$ \\
\hline XJTU & 77 & $\begin{array}{l}\text { Public, Environmental \& Occupational Health(117); Environmental Studies (35); Social Sciences, } \\
\text { Interdisciplinary(21); Economics(20); Information Science \& Library Science(18); } \\
\text { Demography(18); Sociology(18). }\end{array}$ \\
\hline
\end{tabular}

Figure 2 reveals the performance of the top 20 research themes of Huazhong University of Science and Technology in the social science field. As shown in the figure, there are the most papers under the theme of Public, Environmental \& Occupational Health and the citation frequency is highest, but the CNCI value is less than the global average and also lower than the CNCI average value of the papers in the university in this field. There are more papers under the theme of Information Science \& Library Science and the citation frequency is higher, and CNCI is also higher than the average of the university. The most noteworthy is Management, and there are not many papers under this theme is not high, but $\mathrm{CNCI}$ is far higher than other themes. In addition, there are not many papers related to Geography, but the CNCI is higher.

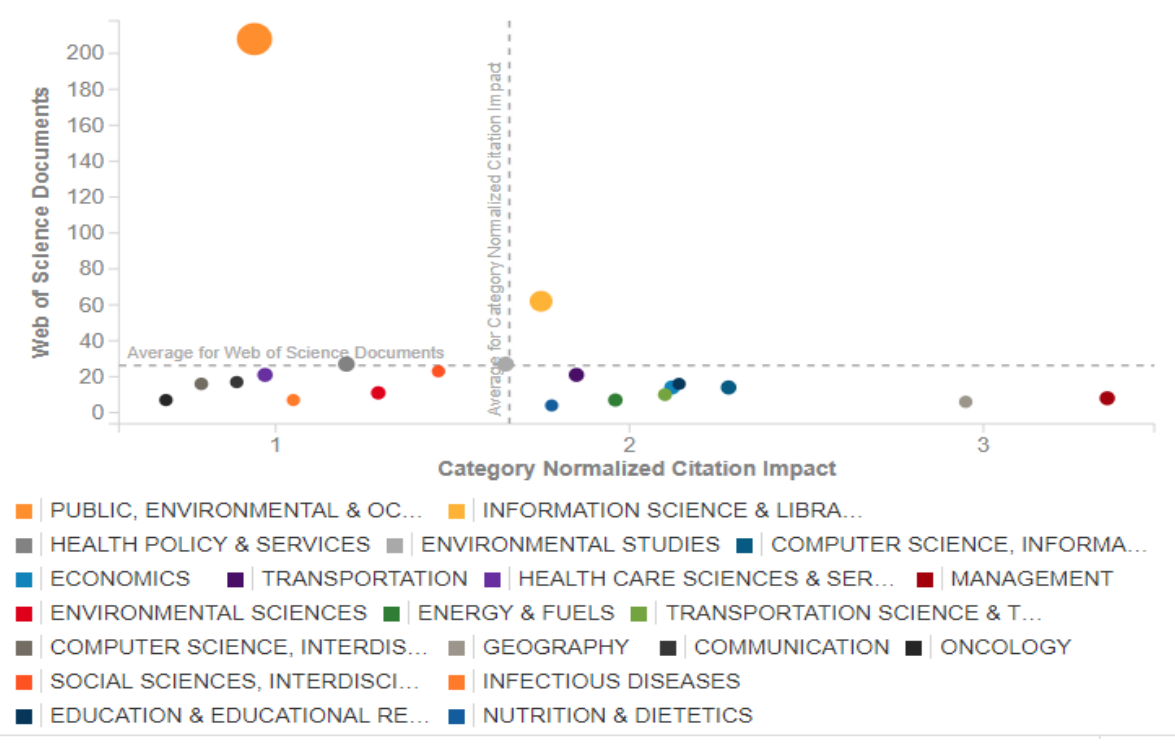

Fig. 1. HUST research theme performance analysis 


\section{E. HUST Scientific Research Collaboration Performance Analysis}

An analysis of the papers of Huazhong University of Science and Technology in the field of social science shows that the university conducted research collaboration with 258 institutions from 2007 to 2017, including 150 international institutions and 108 institutions in China. The 12 papers in collaboration with Univ Hong Kong were cited by 227 time, 28 papers in collaboration with Wuhan University were cited by
223 times, and 13 papers in collaboration with Harvard Univ were cited by 209 times. The CNCI of a paper in collaboration with Univ Rochester was highest, up to 27.58; The CNCI of a paper in collaboration with Jiangxi Sci Tech Norm Univ was as high as 10.76; And The CNCI of a paper in collaboration with Stellenbosch Univ and Western Univ was as high as 6.8.

TABLE V. HUST SCIENTIFIC RESEARCH COLLABORATION PERFORMANCE ANALYSIS

\begin{tabular}{lll}
\hline By Cites(cites) & By papers(papers) & By CNCI(papers, CNCI) \\
\hline Univ Hong Kong(227) & Wuhan Univ(28) & Univ Rochester(1, 27.58) \\
Wuhan Univ(223) & Harvard Univ(13) & Jiangxi Sci Tech Norm Univ(1, 10.76) \\
Harvard Univ(209) & Univ Hong Kong(12) & Royal Melbourne Inst Tech(1, 6.8) \\
City Univ Hong Kong(209) & Univ Texas Sys(12) & Zhejiang Univ(5, 6.47) \\
Univ Texas Sys(155) & Hubei Univ Med(12) & Stellenbosch Univ $(1,6.47)$ \\
\hline
\end{tabular}

\section{F. HUST School and Department Contribution Analysis}

From 2007 to 2017, a total of 31 schools and departments from Huazhong University of Science and Technology had published papers in ESI social science field. The three departments with a relatively large number of publications include the School of Public Health, School of Management and School of Medicine \& Health Management, they also contributed a relatively high citation frequency. Among them, the School of Public Health published the most papers, reaching 140; The School of Management and School of
Medicine \& Health Management had published more than 50 papers, the School of Public Administration and the School of Foreign Languages had published more than 20 papers, Tongji Hospital, Union Hospital, the School of Civil Engineering \& Mechanics and the School of Journalism \& Information Communication had published more than 10 papers, 13 schools such as the School of Automation had published more than 2 papers, and 9 schools and departments such as the School of Software had published 1 paper.

TABLE VI. CONTRIBUTION ANALYSIS OF HUST SCHOOLS AND DEPARTMENTS

\begin{tabular}{lcclcc}
\hline \multicolumn{1}{c}{ Sch/Dept } & Doc & Cites & \multicolumn{1}{c}{ Sch/Dept } & Doc & Cites \\
\hline Sch Public Health & 140 & 1048 & Sch Environmental Sci \& Eng & 5 & 33 \\
Sch Management & 71 & 640 & Sch Life Sci \& Tech & 5 & 19 \\
Sch Med \& Hlth Management & 67 & 458 & Sch Economics & 4 & 21 \\
Sch Public Administration & 28 & 167 & Sch Humanities & 4 & 11 \\
Sch Foreign Languages & 24 & 20 & Sch Energy \& Power Eng & 3 & 41 \\
Tongji Hosp & 18 & 109 & Dept Forensic Medicine & 3 & 4 \\
Wuhan Union Hosp & 13 & 57 & Sch Education & 3 \\
Sch Civil Eng \& Mechanics & 11 & 34 & Sch Hydropower \& Inform Eng & 2 \\
Sch Journalism \& Inform Commun & 11 & 9 & Sch Computer Sci \& Tech & 42 \\
Family Planning Research Inst & 6 & 29 & Liyuan Hosp & 2 & 13 \\
Sch Automation & 6 & 25 & Wuhan Mental Hlth Ctr & 2 & 13 \\
\hline
\end{tabular}




\section{CONCLUSION AND SUGGESTIONS}

Huazhong University of Science and Technology ranks No. 864 in the list of 1,382 institutions in the ESI social sciences, which shows that the university has certain strengths and advantages in the field of social sciences. However, compared with Tsinghua University, Shanghai Jiaotong University and Zhejiang University, there are gaps in the number of papers, international collaboration and journal distribution and there is still much room for improvement. Therefore, the following suggestions are proposed for the construction and development of social sciences in the university:

\section{A. Paying attention to paper output}

The paper is an important expression form of scientific research achievements. The number of papers is one of the commonly used indicators for the scientific research capacity of the evaluation institutions. Some scholars have studied the papers of thirty-eight 985 colleges and universities in the field of social science from 2005 to 2016 and found that the gap between the universities in the number of published papers is gradually widening [7]. From 2007 to 2017, the annual growth rate of Huazhong University of Science and Technology was steady, with only a larger growth rate in 2013. The output of the papers has been lower than that of Tsinghua University, Shanghai Jiaotong University and Zhejiang University and slightly higher than that of Xi'an Jiaotong University since 2013. However, a larger growth rate was occurred in Xi'an Jiaotong University in 2017, and the number of papers has caughted up with that of Huazhong University of Science and Technology.

There is still a large gap between Huazhong University of Science and Technology and the other three universities in the number of papers in the social science. Tsinghua University and Zhejiang University have nearly twice as many papers in this field than Huazhong University of Science and Technology, and Shanghai Jiaotong University is also $40 \%$ higher than Huazhong University of Science and Technology. Huazhong University of Science and Technology should pay attention to the output of the paper, especially to publish more high-level papers.

\section{B. Strengthening international collaboration}

Among the five universities, the number and percentage of internationally collaborated papers in Huazhong University of Science and Technology are lowest. Zhejiang University has the most internationally collaborated papers, up to 395 , and 158 for Huazhong University of Science and Technology. The total number of papers of Huazhong University of Science and Technology is $52.40 \%$ of Zhejiang University, but the number of internationally collaborated papers is only $40.00 \%$ of Zhejiang University. The percentage of internationally collaborated papers of Shanghai Jiaotong University is highest, up to 55.11\%; Huazhong University of Science and Technology is only $36.24 \%$.
The analysis shows that Huazhong University of Science and Technology has established good collaboration with many international agencies and has produced very good results. There are more papers in collaboration with Univ Hong Kong and Harvard Univ and the higher citation frequency is also resulted. There are not many papers in collaboration with Univ Rochester, Stellenbosch Univ and Western Univ, but it has a higher influence. In the future, the collaboration and exchanges with these universities should be further strengthened to produce more results and better results.

\section{Appropriately expanding the scope of research and focusing on targeted development}

Compared with the other four universities, the research scope of Huazhong University of Science and Technology is relatively narrow. The social science papers of the university involve in 69 research themes, the papers with the highest literature volume in Zhejiang University involve in 94 research themes, and about 80 for Tsinghua University and Shanghai Jiaotong University respectively, and the papers with the lowest literature volume in Xi'an Jiaotong University akso involve in 77. In addition, the number of literature generated by different research themes is very unbalanced. Among the research themes involved, Public, Environmental \& Occupational Health ranks the first, under which there are 208 papers, and the number of papers possesses an absolute advantage and the citation frequency is also highest. There are only 62 papers under the second-ranked theme of Information Science \& Library Science.

The analysis of the research performance of 69 themes shows that the number of papers under the theme of Public, Environmental \& Occupational Health is highest and the citation frequency is also highest, but its influence is lower; The number of papers under the theme of Information Science $\&$ Library Science and the citation frequency are performed well, and CNCI is also higher than the average value of the whole university; There are research papers under the theme of Management and Geography are fewer but better, although the number of literature is not much, but the $\mathrm{CNCI}$ is higher. Therefore, it is suggested that the scope of research should be broadened in the future, and focus should be put on the development of potential research projects with good development prospects.

\section{Giving more incentives for schools and departments that have made outstanding contributions}

Huazhong University of Science and Technology has a total of 45 schools and departments [8]. Over the past ten years, 31 schools and departments have published papers in ESI social science field, including both liberal arts and science and engineering departments. The School of Public Health, the School of Management and the School of Medicine \& Health Management made outstanding contributions to this subject. Both of the number of papers and citation frequency rank the top three. The School of Public Administration also contributed more papers and citations, and the papers of Tongji Hospital were also highly cited. Among the arts schools and departments, the number of papers published by the School of Foreign Languages and the School of Journalism \& 
Information Communication were considerable, i.e. 24 papers and 11 papers published respectively. The number of papers published by the School of Hydropower \& Digital Engineering and the School of Energy \& Power Engineering was small, but the citation frequency per paper is higher.

The ESI social science field is more comprehensive subject. Its development requires both the flourishing of arts department and the efforts of the department of science and engineering. The university should support the relevant research directions of some schools and departments, give more incentives to the schools and departments that have made outstanding contributions, encourage them to publish more and better papers in order to promote the construction and development of the subject.

\section{REFERENCES}

[1] ESI. [EB/OL]. [2018-03-16]. https://esi.incites.thomsonreuters.com.

[2] InCites. [EB/OL]. [2018-03-16].https://incites.thomsonreuters.com.

[3] Dong Zheng-e, Chen Huilan. The Discipline Forecast of Donghua University Based on the Databases of ESI and InCites [J], Journal of Donghua University, 2013, 39(5): 689-694.

[4] Zhang Xiaoyang, Wei Heng. International Comparative Study of ESI Social science of Chinese Mainland Universities [J], China Collective Economy, 2015(22):104-108.

[5] ESI Journal List. [EB/OL]. [2018-03-16].http://ipsciencehelp.thomsonreuters.com/inCites2Live/8290-TRS.html.

[6] Zhang Lan, Zhou Biyu, Chen Zhishuo. A study of ESI assessmentoriented measures for the improvement of humanities and social science disciplines [J]. Journal of Zhejiang University of Technology (Social Science), 2017, 16(3):338-342, 347.

[7] Sheng Yiping. The Analysis of the Development Trend in the Field of Social science of 985 Project Universities during 2006 to 2015 [J]. Science and Technology Innovation Herald, 2016(18):124-127.

[8] HUST. Schools and departments. [EB/OL]. [2018-03-16]. http://www.hust.edu.cn/726/list.htm. 